\title{
Study of the Influence of Pore Width on the Disposal of Benzene Employing Tunable OMCs
}

\author{
Gang Wang, Zhongshen Zhang, Junhui Wang, Na Li, and Zhengping Hao* \\ Research Center for Eco-Environmental Sciences, Chinese Academy of Sciences, Beijing 100085, China
}

\begin{abstract}
By employing SBA-15 as a hard template and boric acid as a pore-expanding agent, we synthesized a series of tunable ordered mesoporous carbons (OMCs) with high specific surface areas and large pore volumes. The synthesized OMCs were used as adsorbents for benzene disposal to explore the influence of pore width on the adsorption of benzene. The XRD and nitrogen adsorption results revealed that the pore sizes of OMCs were contracted at certain values, within the range of 3.4-7.7 $\mathrm{nm}$. The adsorption results for benzene on the OMCs showed that there was a gradual decrease in adsorption potential as the pore size of the adsorbent material increased. In addition, an evaluation of the dynamic adsorption of benzene per unit surface area indicated that the adsorption behaviors were strongly influenced by the pore width of the adsorbent material.
\end{abstract}

\section{INTRODUCTION}

Volatile organic compounds (VOCs) are a type of special gas pollutants emitted from different sources and processes. ${ }^{1-3}$ Considering the inherently hazardous effects of VOCs on the environment and human health, the control of VOC emissions has attracted much attention in recent years. ${ }^{4-6}$ Many technologies, such as adsorption, absorption, catalytic oxidation, and biofiltration, have been used in practical applications for the removal of industrial VOCs. ${ }^{6}$ Among these methods, adsorption technology has always been a prominent choice because of its efficiency and relatively low cost, especially in adsorption procedures for recovering high-value adsorbates. $^{5,7-9}$ One of the most important issues in adsorption technology is the choice of proper adsorbent. To date, carbon materials such as activated carbon and activated carbon fiber have been widely used as efficient adsorbents for various types of VOCs. ${ }^{1,10}$ However, the irregularly shaped pores and relatively small pore sizes of such microporous adsorbents lead to pore blocking and a decrease of the adsorption/ desorption rate during adsorption procedures, which limits the further improvement of their adsorption performances. ${ }^{11,12}$ Moreover, to prepare adsorbents with high adsorption efficiencies, further research and studies associated with the adsorption mechanism are still needed.

Ordered mesoporous carbon (OMC) is a type of special carbon material that has regular pore shapes and large specific surface areas and pore volumes and is quite different from traditional carbon adsorbents. ${ }^{13,14}$ Moreover, the pore size distributions of OMCs can be easily tuned by adding some pore-expanding agents or selecting different surfactant templates. ${ }^{15,16}$ Benefiting from these advantages, OMCs can be used as efficient adsorbents and model materials in the adsorption field. ${ }^{17} \mathrm{He}$ and $\mathrm{Hu}^{18}$ investigated the adsorption properties of CMK-3 toward three different commercial anionic dyes. Their results indicated that CMK-3 can be used as an efficient adsorbent for the removal of anionic dyes, with adsorption capacities that are 90-200\% higher than those traditional activated carbons. Saha and Deng ${ }^{19}$ employed OMC doped with metals as potential adsorbents for hydrogen storage and found that the adsorption capacity of hydrogen at $25^{\circ} \mathrm{C}$ and 300 bar on nickel-doped OMC could reach 2.14 wt \%. Recently, Yuan et al. ${ }^{20}$ studied the adsorption and separation properties of $\mathrm{CO}_{2}, \mathrm{~N}_{2}$, and $\mathrm{CH}_{4}$ on $\mathrm{OMC}$. The results revealed that $\mathrm{OMC}$ has high adsorption capacities for $\mathrm{CO}_{2}$ and $\mathrm{CH}_{4}$ and high selectivities for $\mathrm{CO}_{2} / \mathrm{CH}_{4}, \mathrm{CH}_{4} / \mathrm{N}_{2}$, and $\mathrm{CO}_{2} / \mathrm{N}_{2}$ mixtures simultaneously. All of the relevant research results confirm the superior adsorption properties of OMCs. However, to the best of our knowledge, little research has been performed on the adsorption properties of VOCs on OMCs, considering the inherently hazardous properties of VOCs and the urgent need for their disposal. Moreover, in view of adsorbent characteristics that influence adsorption properties, most studies have focused on pore volume whereas few have considered the effects of the pore size distribution of the adsorbent. ${ }^{21,22}$

In this research, benzene was selected as a typical adsorbent for VOCs, and the main purpose of this study was to investigate the adsorption properties of benzene on OMCs with different pore sizes. OMCs were synthesized through the hard template method, using SBA-15 as the hard template and sucrose as the carbon precursor. SBA-15 is a type of mesoporous silica material with ordered concentrated pores, and it was widely used as a hard template because the silica can be removed by $\mathrm{HF}$ or $\mathrm{NaOH}$ solution. ${ }^{23}$ Furthermore, during the synthesis process, boric acid was added as a pore-expanding agent to produce OMCs with tunable pore size distributions without changing the primary pore structure. During the carbonization process, the boric acid is expected to form boron oxide and borosilicate nanolayers between the silica and carbon frameworks. Removal of these compounds leads to the enlargement of the pores in the resultant OMC materials. ${ }^{24}$ The adsorption properties of benzene on OMCs under static and dynamic conditions were systematically studied. Meanwhile, the effects of OMC pore size on the adsorption properties of benzene are also discussed.

Received: September 22, 2014

Revised: December 8, 2014

Accepted: January 6, 2015

Published: January 6, 2015 


\section{EXPERIMENTAL SECTION}

2.1. Materials and Chemicals. Poly(propylene oxide)- $b$ poly(ethylene oxide)- $b$-poly(propylene oxide) triblock copolymer Pluronic 123 was purchased from Sigma-Aldrich. Ethyl silicate $\left(\mathrm{SiO}_{2},>28.4 \%\right)$, hydrochloric acid (36.0-38.0\%), ethanol (>99.7\%), boric acid (>99.8\%), sucrose (>99.0\%), sulfuric acid (95.0-98.0\%), hydrofluoric acid (>40.0\%) were purchased from Sinopharm Chemical Reagent Company. All chemicals were used as received without any purification process. Distilled water was used in all of the experiments.

2.2. Synthesis of OMC. The hard template method was employed to synthesize the OMCs according to the reported procedure with some modifications. ${ }^{23,24}$ During the synthetic procedure, SBA-15 was selected as the hard template, and sucrose was used as the carbon precursor. First, the soft template synthesis method was employed to synthesize siliceous template SBA-15. The triblock copolymer P123 and ethyl silicate were employed as the sources of template and silica, respectively. Typically, $32 \mathrm{~g}$ of Pluronic 123 was first dissolved in $1000 \mathrm{~g}$ of distilled water and $200 \mathrm{~g}$ of $\mathrm{HCl}$, and the mixture was stirred at $40^{\circ} \mathrm{C}$ for $4 \mathrm{~h}$ to form a template solution. Then, $68.8 \mathrm{~g}$ of ethyl silicate was added to the solution with vigorous stirring at $40{ }^{\circ} \mathrm{C}$ for $15 \mathrm{~min}$. After that, the solution was aged at 40 and $100{ }^{\circ} \mathrm{C}$ for $24 \mathrm{~h}$ each. Afterward, the sediment was washed thoroughly with ethanol, and SBA-15 was obtained by calcination at $550{ }^{\circ} \mathrm{C}$ in air for $2 \mathrm{~h}$.

The synthesized mesoporous siliceous SBA-15 material was then used as a template for the synthesis of OMCs. Sucrose and boric acid were used as the carbon source and pore-expanding agent, respectively. In a typical synthesis process, $0.141 \mathrm{~g}$ of sulfuric acid, $1.25 \mathrm{~g}$ of sucrose, and a certain amount of boric acid were dissolved into $5 \mathrm{~mL}$ of distilled water, and then $1 \mathrm{~g}$ of SBA-15 was added to the solution and dispersed by the supersonic method. The resultant pasty sample was dried at 70 ${ }^{\circ} \mathrm{C}$ for $1 \mathrm{~h}$ and then precarbonized at 100 and $160{ }^{\circ} \mathrm{C}$ for $6 \mathrm{~h}$ each. The obtained dark-brown solid sample was then ground into a powder, and the above immersion and carbonization processes were repeated. The quality of the impregnation liquor for the second time was $66 \%$ of that of the first time, with the same mass ratio of sulfuric acid, sucrose, boric acid, and water. The obtained composites were carbonized at $900{ }^{\circ} \mathrm{C}$ for $3 \mathrm{~h}$ under a nitrogen atmosphere at a heating rate of $5{ }^{\circ} \mathrm{C} / \mathrm{min}$. After being cooled to room temperature, the composite was washed with $10 \%$ hydrofluoric acid to remove the SBA-15 template, and then washed with distilled water until neutral $\mathrm{pH}$. Finally, the OMC materials were obtained after being dried at $60{ }^{\circ} \mathrm{C}$ overnight. The OMC samples are denoted as OMC- $x \%$, where $x$ is the molar ratio of boric acid, that is, $x=n$ (boric acid $) /[n$ (boric acid $)+n($ sucrose $)]$, where $n$ represents the number of moles. The values of $x$ used in the work were $x=20$, $35,45,55$, and 75 .

2.3. Material Characterization. Small-angle X-ray diffraction (XRD) patterns were recorded on a PANalytical X'Pert PRO MPD instrument using $\mathrm{Cu} \mathrm{K} \alpha$ radiation $(\lambda=1.540598$ $\AA$ ). High-resolution scanning electron microscopy (HRSEM) analysis was conducted on a JEM 2010 instrument. Texture properties were measured using nitrogen sorption isotherms at liquid nitrogen temperature on a Micromeritics ASAP 2020 gas sorption analyzer. Before measurements, all samples were degassed overnight at $110^{\circ} \mathrm{C}$. The surface area was calculated using Brunauer-Emmett-Teller (BET) theory. The total pore volume was obtained by the nitrogen amount adsorbed at $P / P_{0}$
$=0.99$. The micropore volume was determined by the $t$-plot method. The pore size distributions were calculated by analyzing the desorption branch of the isotherms based on the Barrett-Joyner-Halenda (BJH) model.

2.4. Adsorption Measurements. The static adsorption properties of benzene on the OMCs were measured on a HIDEN Intelligent Gravimetric Analyzer IGA-002. In this work, adsorption isotherms were obtained at 25,35 , and $45^{\circ} \mathrm{C}$. The saturation vapor pressure of the adsorbate was calculated according to the Antoine equation

$$
\log p_{0}=A-\frac{B}{T+C}
$$

where $p_{0}$ is the saturated vapor pressure (Torr); $T$ is the temperature $\left({ }^{\circ} \mathrm{C}\right)$; and $A, B$, and $C$ are constants defined for the adsorbate. For benzene $\left(8-103{ }^{\circ} \mathrm{C}\right)$, the corresponding parameters are as follows: $A=6.90565, B=1211.033$, and $C$ $=220.790 .^{25}$

The dynamic adsorption properties of benzene on the OMCs were measured on a fixed-bed system as described in our previous work. $^{26}$ The gas concentration of benzene was about 650 ppmv. Before adsorption, all of the carbon materials were degassed at $60{ }^{\circ} \mathrm{C}$ for $10 \mathrm{~h}$ to remove the physically adsorbed water molecules and other impurities. The inner diameter of the adsorption column was $6 \mathrm{~mm}$. In each experiment, $50 \mathrm{mg}$ of OMC was loaded, and the total gas flow rate was maintained at $50 \mathrm{~mL} \cdot \mathrm{min}^{-1}$. During the whole experimental procedure, the concentration of benzene at the outlet of the reactor was determined intermittently using a photoionization VOC detector. ${ }^{27}$ The breakthrough time is defined as the time when the outlet concentration was about $5 \%$ of the inlet concentration. ${ }^{28}$ The adsorbed amounts were calculated by the equation

$$
q=\frac{F}{M w}\left(C_{\mathrm{i}} t_{\mathrm{s}}-\int_{0}^{t_{\mathrm{s}}} C_{\mathrm{o}} \mathrm{d} t\right)
$$

where $q$ is the adsorbed amount $\left(\mathrm{mmol} \cdot \mathrm{g}^{-1}\right), \quad F$ is the volumetric flow rate of carrier gas $\left(\mathrm{mL} \cdot \mathrm{min}^{-1}\right), w$ is the net weight of adsorbent $(\mathrm{g}), M$ is the molar mass of benzene ( $\mathrm{g}$. $\left.\mathrm{mol}^{-1}\right), C_{\mathrm{i}}$ represents the benzene concentration at the inlet $(\mathrm{g}$. $\left.\mathrm{mL}^{-1}\right), C_{\mathrm{o}}$ is the benzene concentration at the outlet $\left(\mathrm{g} \cdot \mathrm{mL}^{-1}\right)$, and $t_{\mathrm{s}}$ is the saturation time of adsorbent ( $\mathrm{min}$ ).

The isosteric heats of adsorption are usually used as a measure of the surface heterogeneity of a solid adsorbent. The values can be calculated by the Clausius-Clapeyron equation, which can be written in the form ${ }^{33}$

$$
Q_{\mathrm{st}}=-R\left(\frac{\partial \ln P}{\partial T^{-1}}\right)_{\theta}
$$

where $Q_{\mathrm{st}}$ is the isosteric heat of adsorption at a given uptake $\theta$, $P$ is the vapor pressure, $T$ is the temperature, and $R$ is the gas constant.

\section{RESULTS AND DISCUSSION}

3.1. Characterization of OMC. The XRD patterns of SBA15 and the OMCs are depicted in Figure 1. The three diffraction peaks in the range $0.5-2^{\circ}$ can be indexed to the (100), (110), and (200) reflections of SBA-15 in the dimensional hexagonal space group $p 6 \mathrm{~mm}$, implying the successful synthesis of SBA-15. ${ }^{29}$ All of the OMCs exhibited diffraction peak at $2 \theta=1-1.2^{\circ}$, which corresponds to the twodimensional hexagonal crystal structure (100) diffraction, 


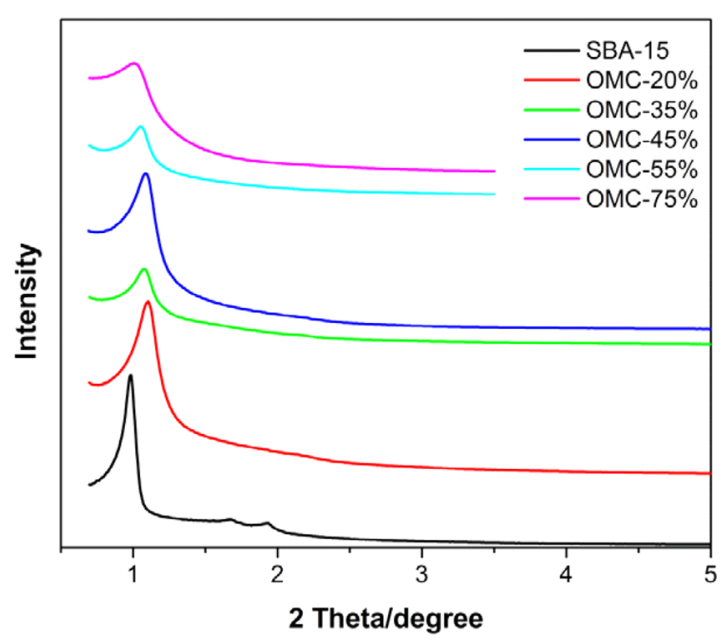

Figure 1. XRD patterns of SBA-15 and OMCs.

indicating that the OMCs reproduced the mesoscopic structure of SBA-15. For these OMCs, there was a slight shift to lower angle for the diffraction peak at $2 \theta=1-1.2^{\circ}$. This might be due to the crescent pore width of the OMCs, caused by the increased dosage of boric acid. In addition, a small diminution of intensity for the characteristic peaks occurred with increasing boric acid dosage, indicating a slight decrease of the long-range order of the OMCs. ${ }^{18}$

To further illustrate the pore structures of the materials, nitrogen adsorption/desorption measurements were employed. The isotherms of SBA-15 and OMCs are the presented in Figure 2. As shown in Figure 2, only small amounts of nitrogen adsorbed on the OMCs in the low relative pressure range, and the adsorbed nitrogen was mainly contributed by mesopores, implying the mesoporous nature of the materials. With an increase in the relative pressure, typical type IV isotherms appeared according to the IUPAC classification, indicating the presence of a concentrated distribution of mesopores. ${ }^{30}$ Moreover, all of the OMCs demonstrated an H1-type hysteresis loop. With increasing boric acid dosage, the OMCs' hysteresis loops shifted to higher relative pressure, indicating a gradual increase in the concentrated mesopores of the OMCs, which agrees well with the XRD results. Additionally, the inflection point of each isotherm where capillary condensation occurs grew gradually to a higher relative pressure value as the dosage of boric acid increased. The pore size distribution curves of the OMCs obtained from the $\mathrm{BJH}$ model are also shown in Figure 2. They are mainly in the range of $2-12 \mathrm{~nm}$, as the larger parts of the pore size can be neglected. The pore size distributions of the OMCs were concentrated at certain widths. The most probable distributed pores were $3.5,4.0,4.5,5.2,6.0$, and $7.0 \mathrm{~nm}$, respectively, when the pore-expanding agent was increased from $20 \%$ to $75 \%$ in sequence. It is clear that an increase in the centralized pore diameter occurred as the amount of pore-expanding agent rose, indicating the successful synthesis of pore-width-tunable OMCs.

The texture properties of the samples are summarized in Table 1 . Generally, the pore volumes and micropore volumes of the OMCs were almost the same. The micropore volume of SBA-15 accounted for about $7 \%$ of the total pore volume; this value is similar to that obtained in previous research, confirming the successful synthesis of SBA-15. ${ }^{31}$ With increasing amount of boric acid, an increase in the average pore diameter was
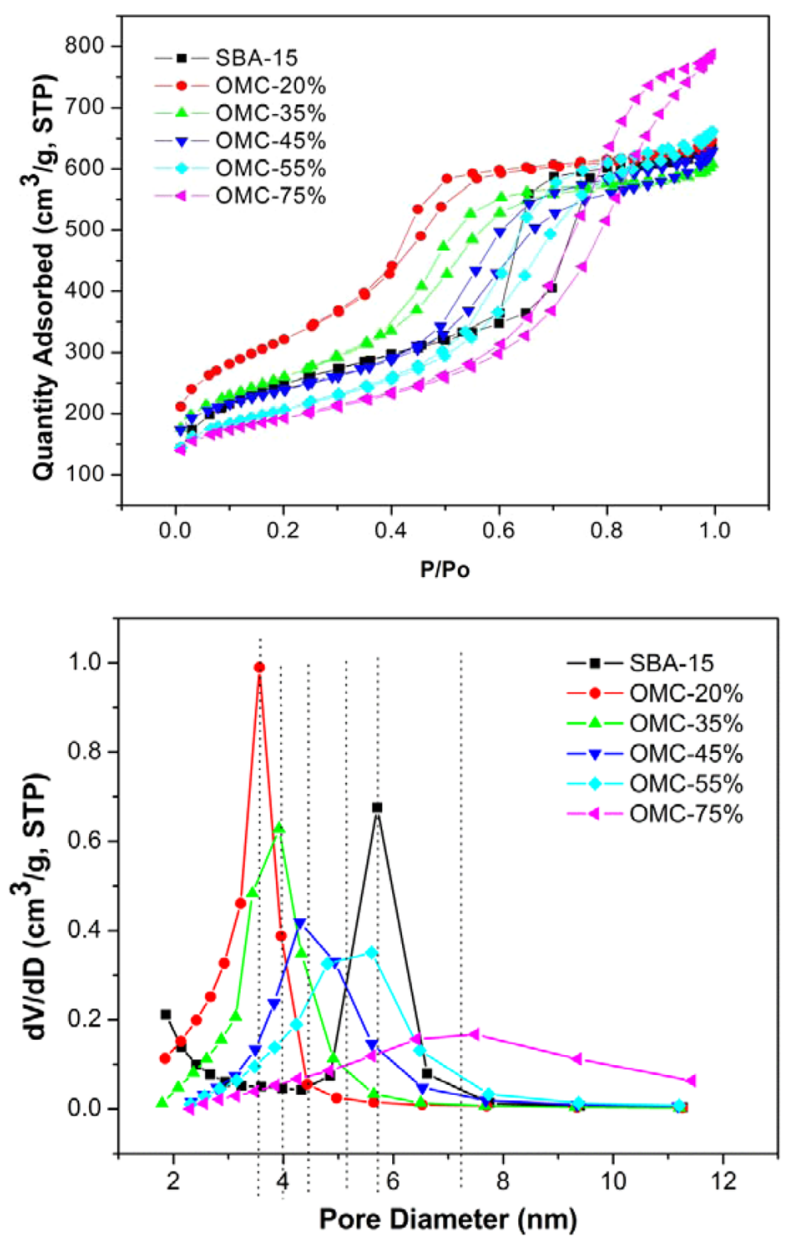

Figure 2. Nitrogen adsorption/desorption isotherms and pore size distribution curves of SBA-15 and OMCs.

Table 1. Texture Properties of SBA-15 and OMCs

\begin{tabular}{lrcccc} 
sample & $\begin{array}{c}\text { surface } \\
\text { area } \\
\left(\mathrm{m}^{2} / \mathrm{g}\right)\end{array}$ & $\begin{array}{c}\text { pore } \\
\text { volume } \\
\left(\mathrm{cm}^{3} / \mathrm{g}\right)\end{array}$ & $\begin{array}{c}\text { micropore } \\
\text { area } \\
\left(\mathrm{m}^{2} / \mathrm{g}\right)\end{array}$ & $\begin{array}{c}\text { micropore } \\
\text { volume } \\
\left(\mathrm{cm}^{3} / \mathrm{g}\right)\end{array}$ & $\begin{array}{c}\text { average } \\
\text { pore size } \\
(\mathrm{nm})\end{array}$ \\
SBA-15 & 844.2 & 0.98 & 134.7 & 0.07 & 5.7 \\
OMC-20\% & 1120.8 & 1.00 & 199.9 & 0.09 & 3.4 \\
OMC-35\% & 893.5 & 0.94 & 210.3 & 0.10 & 3.9 \\
OMC-45\% & 792.7 & 0.97 & 294.1 & 0.15 & 4.9 \\
OMC-55\% & 698.8 & 1.02 & 200.3 & 0.10 & 5.4 \\
OMC-75\% & 640.9 & 1.22 & 223.6 & 0.11 & 7.7 \\
\hline
\end{tabular}

observed, and thus, the specific surface area decreased accordingly. Corresponding data revealed that, as the average pore diameter increased from $3.4 \mathrm{~nm}$ for OMC-20\% to $7.7 \mathrm{~nm}$ for OMC- $75 \%$, the specific surface area decreased by about $42 \%$.

To investigate the pore structure of the materials more intuitively, transmission electron microscopy TEM images of SBA-15 and OMCs were recorded and are shown in Figure 3. Clearly, all of the samples exhibited an ordered twodimensional hexagonal mesoporous structure. Meanwhile, a slight decrease in the long-range ordered structure can be seen, as the amount of boric acid increased, which is consistent with the XRD patterns in Figure 1.

3.2. Static Adsorption of Benzene on OMCs. Adsorption isotherms are important because they can be regarded as the primary source of information on a particular 


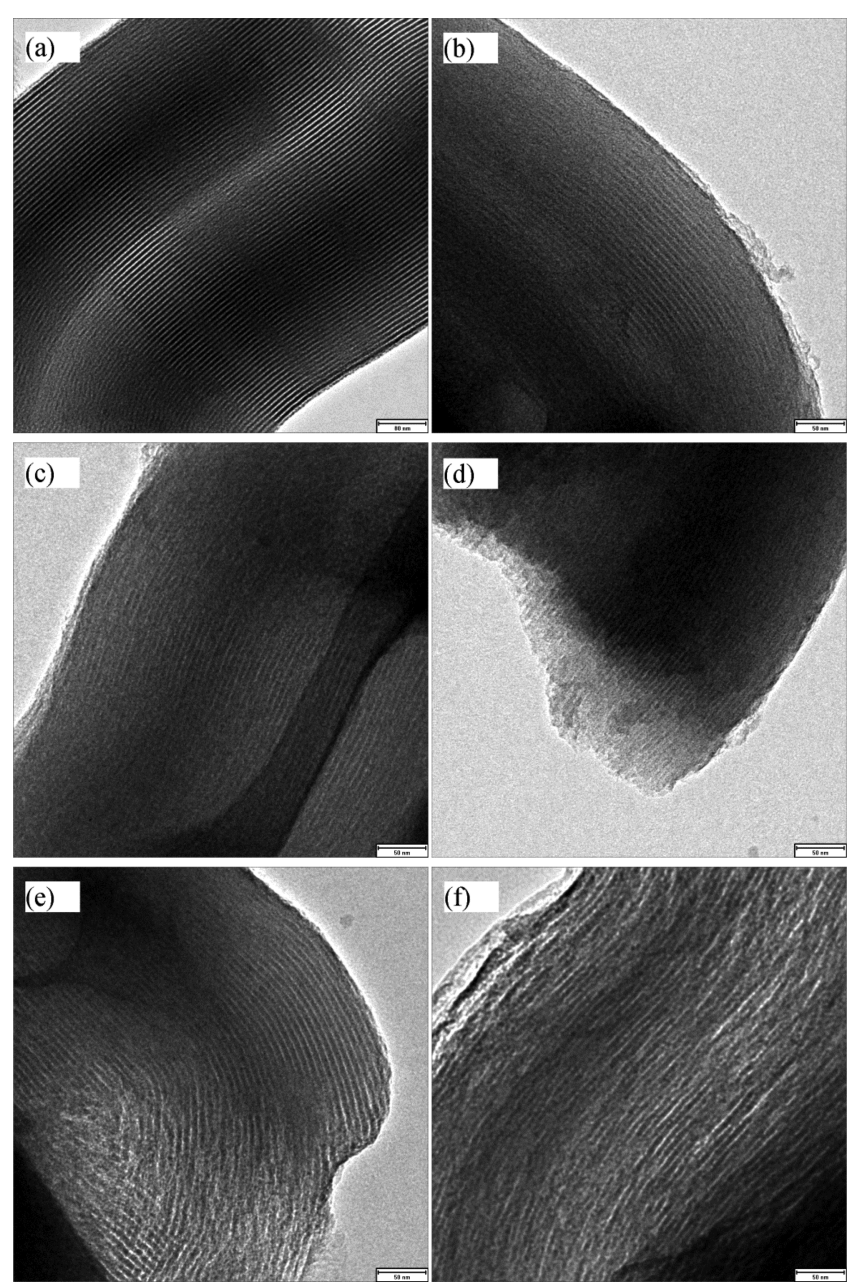

Figure 3. TEM images of (a) SBA-15, (b) OMC-20\%, (c) OMC-35\%, (d) OMC-45\%, (e) OMC-55\%, and (f) OMC-75\%.

adsorbent/adsorbate system and the data can be used to evaluate the adsorption properties. Figure $4 a-e$ shows the adsorption/desorption isotherms of benzene on the OMCs prepared in this work at 25,35 , and $45{ }^{\circ} \mathrm{C}$. Similarly to the nitrogen adsorption/desorption isotherms, each of these benzene adsorption isotherms shows a type IV isotherm and a type $\mathrm{H} 1$ hysteresis loop. The maximum adsorption amounts of benzene on the OMCs at various temperatures are almost the same; this is because the total pore volumes of the OMCs are similar. At the saturation vapor pressure, all of the pores are accessible to the benzene molecule, so similar adsorption amounts are obtained. Moreover, it is interesting to note that, as the temperature was raised from 25 to $45^{\circ} \mathrm{C}$, the adsorption amount of benzene on a single adsorbent at the same vapor pressure decreased. This confirms the physical adsorption characteristics of benzene on the OMCs.

For a more intuitive comparison, all of the adsorption isotherms performed at $25^{\circ} \mathrm{C}$ on the OMCs are gathered in Figure 4f. A clear difference can be seen in that, as the dosage of boric acid increased, the adsorption isotherm of benzene gradually moved to the right; that is, the pore size distributions of the OMCs have a significant influence on the adsorption properties of benzene. Choosing the adsorption amount of benzene at a fixed pressure for comparison, the corresponding value decreased as the pore size increased, except for a small deviation occurring between the isotherms of OMC- $45 \%$ and
OMC-55\%. Previous studies associated with the adsorption properties of VOCs on carbon materials pointed out that, in the case of physical adsorption, the adsorption amount of adsorbate is determined only by the total pore volume and not the pore size distribution. ${ }^{32}$ However, this result is inconsistent with the previous result that, under physical adsorption conditions, the pore size of the adsorbent has a significant influence on the adsorption amounts of adsorbates.

3.3. Isoteric Heats of Adsorption. The corresponding $Q_{s t}$ values of benzene on the OMCs were calculated and are shown in Figure 5. Sample OMC-20\% exhibits the highest isosteric heats of adsorption, especially in the beginning of the adsorption process. In comparison with that of OMC-20\%, the variations of the isosteric heats of the other OMCs seem to be less obvious, because the curves are more stable. Considering that the pore diameter of OMC-20\% is much smaller than those of the other OMCs, it can be concluded that OMCs with narrower pore widths are more accessible for benzene. In addition, although the isosteric heats of OMC-35\%, OMC- $45 \%$, and OMC-55\% are close to each other, it can be concluded that, overall, the isosteric heat decreased with increasing pore size, as the values of the isosteric heat for OMC-20\% are the highest and the corresponding values for OMC-75\% are the lowest. Thus, one can speculate that an adsorbent with a narrower pore diameter has a higher adsorption potential for the benzene adsorption, that is, that benzene molecules are more likely to be adsorbed onto an adsorbent with a narrower pore diameter.

3.4. Dynamic Adsorption of Benzene on OMCs. In practical applications, adsorbents are usually loaded in a fixedbed apparatus and used for adsorption. Thus, the dynamic adsorption of benzene vapor onto the OMCs was performed to simulate the real situation, and the results are shown in Figure 6. As can be seen from Figure 6, there is a clear regularity in dynamic breakthrough curves among the various OMCs. For the OMCs with larger average pore diameters, the breakthrough times are correspondingly shorter. Generally, the longer the breakthrough time is, the higher the dynamic adsorption capacity of an adsorbent will be. Thus, the gradually increased breakthrough time from OMC- $75 \%$ to OMC- $20 \%$, implies higher adsorption amounts of benzene in the same sequence. The dynamic breakthrough times and adsorption amounts of benzene in the OMCs are reported in Table 2. As the pore size of the OMCs increased from 3.4 to $7.7 \mathrm{~nm}$, the breakthrough time decreased from 32 to $6 \mathrm{~min}$, and the corresponding adsorption amount decreased from 0.114 to $0.041 \mathrm{mmol} / \mathrm{g}$.

It is meaningful to reveal how the microscopic structure of an adsorbent is reflected in the macroscopic characteristics of adsorption by employing a single type of adsorbent. In this study, the prepared OMCs had similar total pore volumes and micropore volumes, but the tunable pore sizes and BET specific surface areas exhibited greater variations (the BET specific surface area decreased from 1120.8 to $640.9 \mathrm{~m}^{2} / \mathrm{g}$ ). To eliminate the influence of the surface area and pore volume, normalized adsorption amounts, namely, the adsorption amounts per unit surface area and unit pore volume, were determined (see Table 2). The trend that the dynamic adsorption capacity decreased with the enlargement of the pore size still holds. Furthermore, the amounts adsorbed per unit surface area and per unit pore volume for different samples are depicted in Figure 7, to reveal the relationships between adsorption characteristics and the pore width more intuitively. 

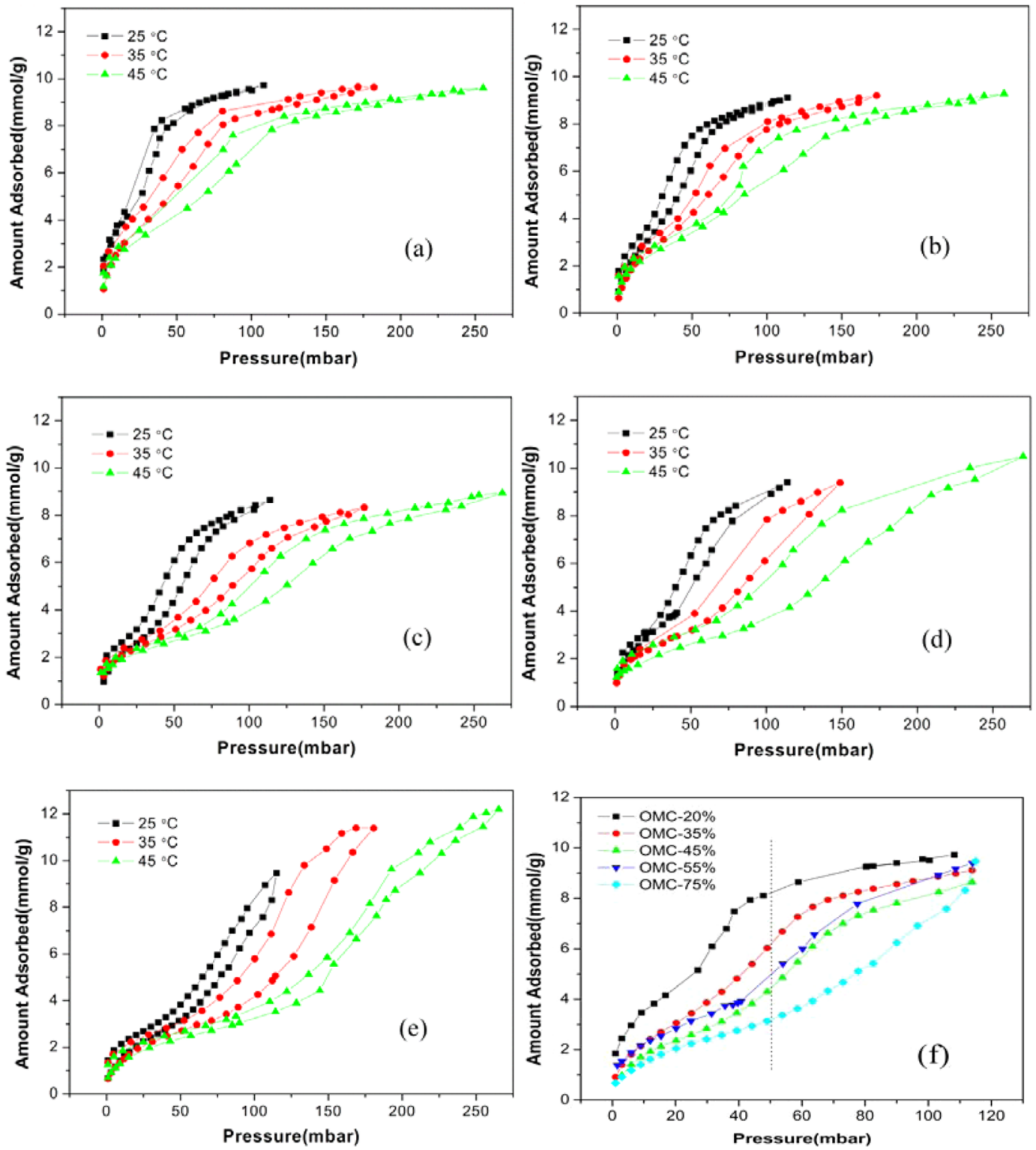

Figure 4. Adsorption isotherms of benzene vapor on (a) OMC-20\%, (b) OMC-35\%, (c) OMC-45\%, (d) OMC-55\%, and (e) OMC-75\% at 25, 35, and $45{ }^{\circ} \mathrm{C}$ and (f) collective adsorption isotherms of benzene at $25{ }^{\circ} \mathrm{C}$ on OMCs.

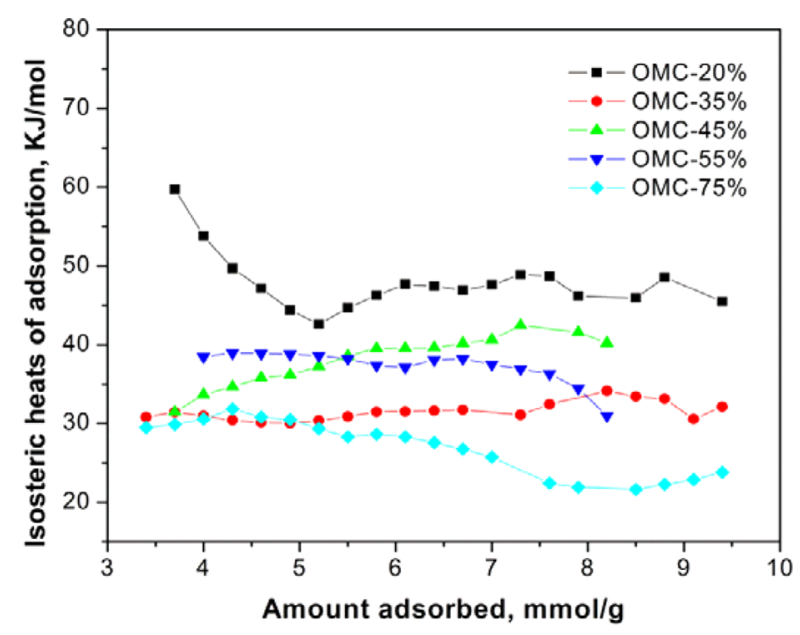

Figure 5. Variation of isosteric heats for benzene adsorption onto OMCs.

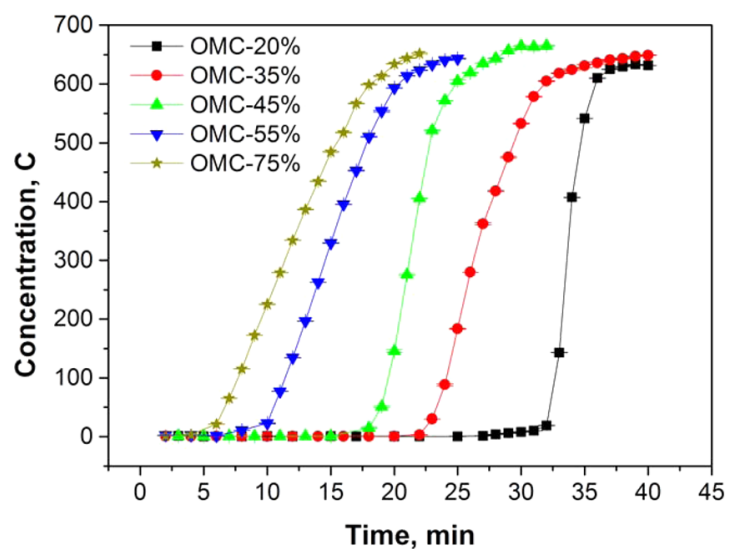

Figure 6. Experimental dynamic breakthrough curves of benzene vapor onto OMCs. 
Table 2. Breakthrough Times and Dynamic Adsorbed Amounts of Benzene Vapor onto OMCs

\begin{tabular}{ccccc} 
sample & $\begin{array}{c}\text { amount } \\
\text { breakthrough } \\
\text { time (min) }\end{array}$ & $\begin{array}{c}\text { adsorbed } \\
(\mathrm{mmol} / \mathrm{g})\end{array}$ & $\begin{array}{c}\mathrm{Q}_{\text {area }}{ }^{2} \\
\left(\mu \mathrm{mol} / \mathrm{m}^{2}\right)\end{array}$ & $\begin{array}{c}\mathrm{Q}_{\text {vol }}{ }^{b} \\
\left(\mathrm{mmol} / \mathrm{cm}^{3}\right)\end{array}$ \\
\hline OMC-20\% & 32 & 0.114 & 0.102 & 0.114 \\
OMC-35\% & 23 & 0.089 & 0.100 & 0.095 \\
OMC-45\% & 18 & 0.073 & 0.092 & 0.075 \\
OMC-55\% & 10 & 0.052 & 0.074 & 0.051 \\
OMC-75\% & 6 & 0.041 & 0.064 & 0.034
\end{tabular}

${ }^{a} Q_{\text {area }}=$ amount adsorbed per unit surface area $\left(\mu \mathrm{mol} / \mathrm{m}^{2}\right) .{ }^{b} Q_{\mathrm{vol}}=$ amount adsorbed per unit pore volume $\left(\mathrm{mmol} / \mathrm{cm}^{3}\right)$.

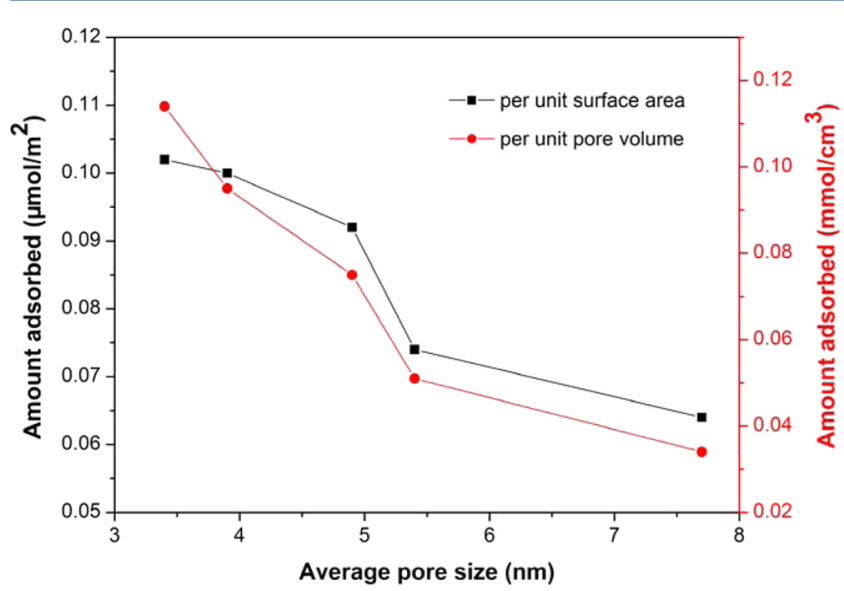

Figure 7. Dynamic adsorption capacities of benzene per unit surface area and unit pore volume on different samples.

This figure helps to conceptualize the relationship between pore width and dynamic adsorption capacity. Clearly, the benzene adsorption amounts per unit surface area and pore volume decreased with increasing average pore diameters of the OMCs. Thus, this figure is able to clarify the relationships between the adsorption characteristics and the textural property unequivocally that, in the mesoporous range, a narrower pore size effectively increases the utilization of the adsorbent's surface area and pore volume. In a word, the pore size distribution is the dominant factor that influences the adsorption characteristics of benzene on the OMCs.

\section{CONCLUSIONS}

In this work, OMCs were synthesized by a hard template method, and boric acid was added during the synthetic procedure to tune the pore sizes in the small mesopore range of 3-8 $\mathrm{nm}$ without changing the main texture of the OMCs. The resultants OMCs were used as adsorbents to evaluate their adsorption properties for benzene. A study of the isosteric heats of adsorption revealed that the OMCs with narrower pore sizes have higher adsorption potentials. The static and dynamic adsorption amounts of benzene no the OMCs indicate that the pore size distribution is the dominant factor influencing the adsorbed amounts of benzene and that, in the mesoporous range, a narrower average pore size is preferred for adsorption and results in a higher adsorbed amount.

\section{AUTHOR INFORMATION}

\section{Corresponding Author}

*Tel.: +86-10-62923564. E-mail: zpinghao@rcees.ac.cn.

\section{Notes}

The authors declare no competing financial interest.

\section{ACKNOWLEDGMENTS}

We gratefully acknowledge financial support from the National High Technology Research and Development Program of China (2012AA063101), the Key Program of National Natural Science Foundation of China (21337003), the Strategic Priority Research Program (XDB05050200), the Science Promotion Program of the Research Center for Eco-environmental Sciences (YSW2013B05), and the Knowledge Innovation Program of Cooperative Interaction Team of the Chinese Academy of Sciences.

\section{REFERENCES}

(1) Tefera, D. T.; Hashisho, Z.; Philips, J. H.; Anderson, J. E.; Nichols, M. Modeling competitive adsorption of mixtures of volatile organic compounds in a fixed-bed of beaded activated carbon. Environ. Sci. Technol. 2014, 48, 5108.

(2) Hernández, M. A.; Velasco, J. A.; Asomoza, M.; Solis, S.; Rojas, F.; Lara, V. H.; Portillo, R.; Salgado, M. A. Alkane adsorption on microporous $\mathrm{SiO}_{2}$ substrata. 1. Textural characterization and equilibrium. Energy Fuels 2003, 17, 262.

(3) Gironi, F.; Piemonte, V. VOCs removal from dilute vapour streams by adsorption onto activated carbon. Chem. Eng. J. 2011, 172, 671.

(4) Wang, S. B.; Ang, H. M.; Tade, M. O. Volatile organic compounds in indoor environment and photocatalytic oxidation: State of the art. Environ. Int. 2007, 33, 694.

(5) Parmar, G. R.; Rao, N. N. Emerging Control Technologies for Volatile Organic Compounds. Crit. Rev. Environ. Sci. Technol. 2009, 39, 41.

(6) He, C.; Xu, L. L.; Yue, L.; Chen, Y. T.; Chen, J. S.; Hao, Z. P. Supported Nanometric Pd Hierarchical Catalysts for Efficient Toluene Removal: Catalyst Characterization and Activity Elucidation. Ind. Eng. Chem. Res. 2012, 51, 7211.

(7) Serrano, D. P.; Calleja, G.; Botas, J. A.; Gutierrez, F. J. Adsorption and hydrophobic properties of mesostructured MCM-41 and SBA-15 materials for volatile organic compound removal. Ind. Eng. Chem. Res. 2004, 43, 7010.

(8) Guillemot, M.; Mijoin, J.; Mignard, S.; Magnoux, P. Adsorption of tetrachloroethylene on cationic X and Y zeolites: Influence of cation nature and of water vapor. Ind. Eng. Chem. Res. 2007, 46, 4614.

(9) Manjare, S. D.; Ghoshal, A. K. Studies on adsorption of ethyl acetate vapor on activated carbon. Ind. Eng. Chem. Res. 2006, 45, 6563.

(10) Wang, H.; Jahandar Lashaki, M.; Fayaz, M.; Hashisho, Z.; Philips, J. H.; Anderson, J. E.; Nichols, M. Adsorption and desorption of mixtures of organic vapors on beaded activated carbon. Environ. Sci. Technol. 2012, 46, 8341.

(11) Ji, L. L.; Liu, F. L.; Xu, Z. Y.; Zheng, S. R.; Zhu, D. Q. Adsorption of Pharmaceutical Antibiotics on Template-Synthesized Ordered Micro- and Mesoporous Carbons. Environ. Sci. Technol. 2010, 44, 3116.

(12) Dou, B.; Li, J.; Hu, Q.; Ma, C.; He, C.; Li, P.; Hu, Q.; Hao, Z.; Qiao, S. Hydrophobic micro/mesoporous silica spheres assembled from zeolite precursors in acidic media for aromatics adsorption. Microporous Mesoporous Mater. 2010, 133, 115.

(13) Ma, T. Y.; Liu, L.; Yuan, Z. Y. Direct synthesis of ordered mesoporous carbons. Chem. Soc. Rev. 2013, 42, 3977.

(14) Li, W.; Zhao, D. Y. An overview of the synthesis of ordered mesoporous materials. Chem. Commun. 2013, 49, 943.

(15) Ryoo, R.; Joo, S. H.; Kruk, M.; Jaroniec, M. Ordered mesoporous carbons. Adv. Mater. 2001, 13, 677.

(16) Deng, Y. H.; Cai, Y.; Sun, Z. K.; Gu, D.; Wei, J.; Li, W.; Guo, X. H.; Yang, J. P.; Zhao, D. Y. Controlled Synthesis and Functionalization of Ordered Large-Pore Mesoporous Carbons. Adv. Funct. Mater. 2010, 20,3658 . 
(17) Wu, Z. X.; Zhao, D. Y. Ordered mesoporous materials as adsorbents. Chem. Commun. 2011, 47, 3332.

(18) He, C.; Hu, X. J. Anionic Dye Adsorption on Chemically Modified Ordered Mesoporous Carbons. Ind. Eng. Chem. Res. 2011, 50, 14070 .

(19) Saha, D.; Deng, S. G. Hydrogen Adsorption on Ordered Mesoporous Carbons Doped with Pd, Pt, Ni, and Ru. Langmuir 2009, 25,12550 .

(20) Yuan, B.; Wu, X. F.; Chen, Y. X.; Huang, J. H.; Luo, H. M.; Deng, S. G. Adsorption of $\mathrm{CO}_{2}, \mathrm{CH}_{4}$, and $\mathrm{N}_{2}$ on Ordered Mesoporous Carbon: Approach for Greenhouse Gases Capture and Biogas Upgrading. Environ. Sci. Technol. 2013, 47, 5474.

(21) Lillo-Ródenas, M. A.; Cazorla-Amorós, D.; Linares-Solano, A. Behaviour of activated carbons with different pore size distributions and surface oxygen groups for benzene and toluene adsorption at low concentrations. Carbon 2005, 43, 1758.

(22) Fairen-Jimenez, D.; Carrasco-Marin, F.; Moreno-Castilla, C. Adsorption of benzene, toluene, and xylenes on monolithic carbon aerogels from dry air flows. Langmuir 2007, 23, 10095.

(23) Wang, H.; Lam, F. L. Y.; Hu, X. J.; Ng, K. M. Ordered mesoporous carbon as an efficient and reversible adsorbent for the adsorption of fullerenes. Langmuir 2006, 22, 4583.

(24) Zhou, J.; Xing, W.; Zhuo, S. P.; Zhao, Y. Capacitive performance of ordered mesoporous carbons with tunable porous texture in ionic liquid electrolytes. Solid State Sci. 2011, 13, 2000.

(25) Fletcher, A. J.; Yüzak, Y.; Thomas, K. M. Adsorption and desorption kinetics for hydrophilic and hydrophobic vapors on activated carbon. Carbon 2006, 44, 989.

(26) Hu, Q.; Li, J. J.; Hao, Z. P.; Li, L. D.; Qiao, S. Z. Dynamic adsorption of volatile organic compounds on organofunctionalized SBA-15 materials. Chem. Eng. J. 2009, 149, 281.

(27) Lashaki, M. J.; Fayaz, M.; Wang, H. H.; Hashisho, Z.; Philips, J. H.; Anderson, J. E.; Nichols, M. Effect of adsorption and regeneration temperature on irreversible adsorption of organic vapors on beaded activated carbon. Environ. Sci. Technol. 2012, 46, 4083.

(28) Britt, D.; Tranchemontagne, D.; Yaghi, O. M. Metal-organic frameworks with high capacity and selectivity for harmful gases. Proc. Natl. Acad. Sci. U.S.A. 2008, 105, 11623.

(29) Hartmann, M.; Vinu, A.; Chandrasekar, G. Adsorption of vitamin $\mathrm{E}$ on mesoporous carbon molecular sieves. Chem. Mater. 2005, 17,829 .

(30) Sing, K. S. W. Reporting physisorption data for gas/solid systems with special reference to the determination of surface area and porosity (Recommendations 1984). Pure Appl. Chem. 1985, 57, 603.

(31) Silvestre-Albero, A.; Jardim, E. O.; Bruijn, E.; Meynen, V.; Cool, P.; Sepulveda-Escribano, A.; Silvestre-Albero, J.; Rodriguez-Reinoso, F. Is There Any Microporosity in Ordered Mesoporous Silicas? Langmuir 2009, 25, 939.

(32) Kosuge, K.; Kubo, S.; Kikukawa, N.; Takemori, M. Effect of pore structure in mesoporous silicas on VOC dynamic adsorption/ desorption performance. Langmuir 2007, 23, 3095.

(33) Wu, H.; Simmons, J. M.; Liu, Y.; Brown, C. M.; Wang, X. S.; Ma, S.; Peterson, V. K.; Southon, P. D.; Kepert, C. J.; Zhou, H. C.; Yildirim, T.; Zhou, W. Metal-Organic Frameworks with Exceptionally High Methane Uptake: Where and How is Methane Stored? Chem.Eur. J. 2010, 16, 5205. 